\title{
Control of hole localization in magnetic semiconductors by axial strain
}

\author{
Hannes Raebiger, ${ }^{1,2,3, *}$ Soungmin Bae, ${ }^{1,2}$ Carlos Echeverría-Arrondo, ${ }^{3,4}$ and Andrés Ayuela ${ }^{3,4}$ \\ ${ }^{1}$ Department of Physics, Yokohama National University, Yokohama, Japan \\ ${ }^{2}$ Centro de Ciências Naturais e Humanas, Universidade Federal do ABC, Santo André, SP, Brazil \\ ${ }^{3}$ Donostia International Physics Centre (DIPC), San Sebastián/Donostia, Spain \\ ${ }^{4}$ Materials Physics Center CFM-MPC Centro Mixto CSIC-UPV/EHU, Department of Materials Physics, San Sebastián/Donostia, Spain
}

(Received 10 September 2017; revised manuscript received 29 November 2017; published 8 February 2018)

\begin{abstract}
Mn and Fe-doped GaN are widely studied prototype systems for hole-mediated magnetic semiconductors. The nature of the hole states around the Mn and Fe impurities, however, remains under debate. Our self-interaction corrected density-functional calculations show that the charge neutral $\mathrm{Mn}^{0}$ and positively charged $\mathrm{Fe}^{+}$impurities have symmetry-broken $d^{5}+h$ ground states, in which the hole is trapped by one of the surrounding $\mathrm{N}$ atoms in a small polaron state. We further show that both systems also have a variety of other $d^{5}+h$ configurations, including symmetric, delocalized states, which may be stabilized by axial strain. This finding opens a pathway to promote long-range hole-mediated magnetic interactions by strain engineering and clarifies why highly strained thin-films samples often exhibit anomalous magnetic properties.
\end{abstract}

DOI: 10.1103/PhysRevMaterials.2.024402

\section{INTRODUCTION}

While hopes for room temperature ferromagnetism of Mn or Fe-doped III-V semiconductors have largely vanished [1], they emerge as model systems to study hole-mediated ferromagnetism and spintronics [2,3]. Hole localization governs the strength, nature, and range of magnetic interactions: extended (host-band-like) states are associated with long-range ferromagnetic interactions, whereas localized (impurity-bandlike) states usually lead to strong short-range interactions. For dilute systems where long-range interactions are crucial, hole localization or trapping usually is detrimental to long-range hole-mediated ferromagnetic interactions [4-6]. To assess whether or not the impurity simply captures carrier holes or electrons by changing its hybridization, one needs to identify the $d^{n}$ configuration or oxidation state [7] of the transition metal impurity. If the impurity itself does not capture carriers, we still need to consider the possibility of carrier trapping at other sites [8].

The electronic configuration of $\mathrm{Mn}$ in $\mathrm{GaN}$ has been studied extensively. In n-type $\mathrm{GaN}, \mathrm{Mn}$ is in its singly negative charge state $\left(\mathrm{Mn}^{-}\right.$, or $\mathrm{Mn}^{+\mathrm{II}}$ oxidation state) and has the $d^{5}$ configuration. Fe has the same $d^{5}$ configuration in its charge neutral state $\left(\mathrm{Fe}^{0}\right.$, or $\mathrm{Fe}^{+\mathrm{III}}$ oxidation state). Under p-type doping, however, $\mathrm{Mn}$ is in the charge neutral state $\left(\mathrm{Mn}^{0}\right)$, and $\mathrm{Fe}$ in the singly positive charge state $\left(\mathrm{Fe}^{+}\right)$, but their oxidation states or $d^{n}$ configurations remain debated. Are these $\mathrm{Mn}^{0}$ and $\mathrm{Fe}^{+}$states best described by $d^{4}$ or $d^{5}+h$ configurations? For $\mathrm{Mn}^{0}$, earlier theories suggest either a Jahn-Teller distorted $d^{4}$ configuration $[9,10]$ or a $d^{5}+h$ configuration with the hole tightly bound in a state similar to the Zhang-Rice polaron [1115], but experiments remain inconclusive on the nature of the $\mathrm{Mn}^{0}$ electronic configuration $[2,16,17]$. For Fe in GaN, even

*hannes@ynu.ac.jp the existence of the $\mathrm{Fe}^{+}$charge state remains unclear $[14,18-$ 20].

We show in this paper that around $\mathrm{Mn}$ or Fe impurities in $\mathrm{GaN}$, hole carriers exhibit a variety of both trapped and delocalized states. We further show that by applying axial strain, the relative stability of the different hole states can be engineered, viz., carrier holes in unstrained GaN tend to be trapped by $\mathrm{Mn}$ or $\mathrm{Fe}$ impurities, but under tensile strain along the wurtzite $c$ axis, delocalized hole states may be stabilized. Thus, the electronic as well as the ensuing magnetic properties of $\mathrm{Mn}$ or Fe-doped $\mathrm{GaN}$ can be engineered by axial strain due to, e.g., interfacial strain, mechanical compression, or the piezoelectric effect. This discovery sheds light on the possible origin of starkly different magnetic properties reported in Mn-doped GaN thin films [21,22] and deepens our understanding of the recently observed piezoelectromagnetic effect in Mn-doped GaN [23].

\section{METHODS}

So far, the theoretical study of the localization of carrier states in magnetic semiconductors has suffered from systematic failures of the different approximations to the density-functional theory [1]. The three basic failures are (i) misplacement of transition metal $d$ levels with respect to band edges, (ii) band gap error and spurious mixing of $d$ levels with host states, and (iii) incapability to localize holes or electrons in polaronic states. For transition metal impurities in semiconductors or insulators, not even hybrid functionals [24] or the $G W$ approximation [25,26] offer systematic corrections to all three failures without additional empirical parameters. In the present paper, we employ a $+U$ correction to accurately predict the transition metal $d$ levels with respect to the GaN valence states $[10,27]$ together with a self-interaction correction (SIC) on the nitrogen $p$ levels, introduced to satisfy the generalized Koopmans condition [28,29]. 
We calculate the electronic structure of $\mathrm{Mn}$ and Fe-doped $\mathrm{GaN}$ in wurtzite supercells of 72 and 96 atoms. We replace one $\mathrm{Ga}$ cation by either a $\mathrm{Mn}$ or $\mathrm{Fe}$ impurity, the doping concentration thus being $2.1 \%$ and $2.8 \%$, respectively. We use the projector augmented-wave method [30] implemented in the VASP code [31] with the GGA-PBE functional, on-site $+U$ corrections of 3.9 and $3.5 \mathrm{eV}$ for $\mathrm{Mn}$ and $\mathrm{Fe} d$ levels, respectively, and $J$ fixed at $1.0 \mathrm{eV}$ [32]. These $+U$ parameters assure a correct description of the thermochemical stability of $\mathrm{Mn}$ and Fe in their different oxidation states [32] and agree well with values widely used in the literature [33]. The calculations are carried out with a plane-wave cutoff energy of $300 \mathrm{eV}$ and a $4 \times 4 \times 4 \vec{k}$ mesh for Brillouin-zone integrations. Structural relaxations are carried out such that forces acting on individual nuclei are $<0.02 \mathrm{eV} / \AA$. We then apply a hole-state potential on the $\mathrm{N} p$ levels such that the generalized Koopmans condition is fulfilled [28,29], yielding $\lambda$ parameters of 5.1 and $3.9 \mathrm{eV}$ for the $\mathrm{Mn}$ and Fe-doped systems, respectively. Notice that these $\lambda$ are larger than $3.4 \mathrm{eV}$ obtained for $\mathrm{Mg}$ doped GaN [29], likely due to the $p-d$ hybridization in GaN:Mn and GaN:Fe; even though the $\lambda$ parameters are system specific [34], the physical properties (charge density distribution, structural relaxation, magnetic moment) hardly vary with $\lambda$ once it exceeds a critical value [28]. This theory has also, for the first time, correctly described the transition from extended to localized carrier states underlying a critical metal-insulator transition in a doped semiconductor [8]. For the present systems, this method accurately reproduces experimentally observed electronic and optical transitions (see Appendix), which further ensures the reliability of the present method for various total energy differences.

\section{GROUND STATE OF THE ISOLATED DOPANT}

We first investigate an isolated impurity, calculated in a supercell of 96 atoms, corresponding to the doping concentration of $\sim 2 \%$. Our calculation shows that the ground state of both $\mathrm{Mn}^{0}$ and $\mathrm{Fe}^{+}$in p-type $\mathrm{GaN}$ is a small polaron in $d^{5}+h$ configuration, with the hole tightly bound to one of the nitrogen atoms next to the impurity, i.e., instead of $\mathrm{Mn}^{0}$ assuming the + III oxidation state, or $\mathrm{Fe}^{+}$the +IV state, one of the nitrogen atoms is oxidized to the + II state. Such small polaron ground states have previously been observed around full or empty $d$ shell impurities, e.g., $\mathrm{Mg}\left(d^{0}\right), \mathrm{Be}\left(d^{0}\right)$, or $\mathrm{Zn}\left(d^{10}\right)$ impurities in the same host [35-38], but never before around a half-filled $d^{5}$ shell.

The density of states for $\mathrm{Mn}^{0}$ and $\mathrm{Fe}^{+}$in 96 atom supercells are given in Figs. 1(a)-1(d). The gap states show $p$ - $d$ hybridization, as described in Ref. [7]. The main difference between $\mathrm{Mn}^{0}$ and $\mathrm{Fe}^{+}$is that $\mathrm{Mn}^{0}$ has a clear crystal field resonance (CFR) state in the gap with a strong $d$ character, whereas for $\mathrm{Fe}^{+}$, the gap states are mostly contributed by $\mathrm{N} p$ states, since the occupied $\mathrm{Fe} d$ levels are well below the valence band maximum $E_{v}$. The $\mathrm{Mn}^{0} \mathrm{CFR}$ states were initially described as a partially occupied triply degenerate state of $t$ representation, typical from the host symmetry [39-41], but here we find a doubly occupied $e$ level clearly separated from an empty $a$ level, as shown in Figs. 1(a) and 1(b). This splitting is associated with a broken-symmetry geometry, already described in Refs. $[9,10]$. Despite their correct description of the geometry, these recent works characterize the $a$ level as mostly Mn $d$ like. This $a$
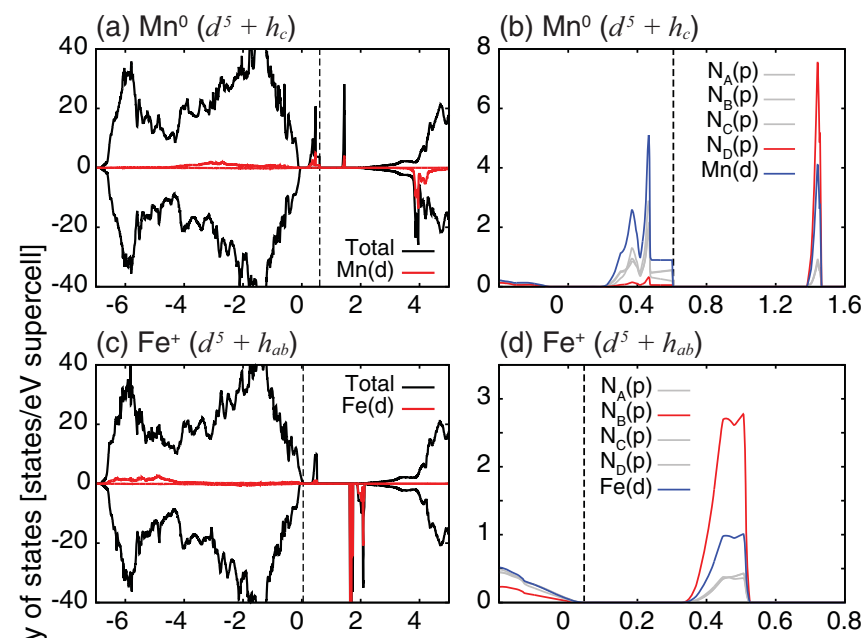

(d) $\mathrm{Fe}^{+}\left(d^{5}+h_{a b}\right)$
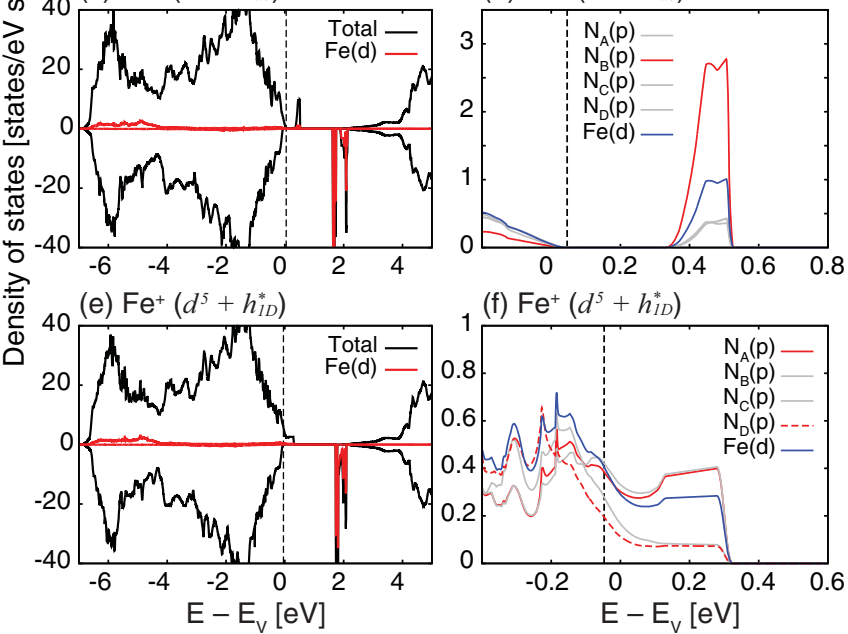

FIG. 1. Densities of states (DOS) of $\mathrm{Mn}^{0}\left(d^{5}+h_{c}\right)$ and $\mathrm{Fe}^{+}\left(h_{a b}^{*}\right)$ ground state configurations in GaN calculated in 96 atom supercells. Panels (a), (c), and (e) show total densities of states and projection on dopant $d$ orbitals. Panels (b), (d), and (f) show orbital projections onto the four neighboring $\mathrm{N}$ atom $p$ orbitals $\left[\mathrm{N}_{\mathrm{A}}(p) \ldots \mathrm{N}_{\mathrm{D}}(p)\right]$ and the dopant $d$ orbitals $[\mathrm{Mn}(d)$ or $\mathrm{Fe}(d)]$ magnified around the highest occupied level indicated by the vertical dashed line. Panels (e) and (f) give the total and projected DOS of the delocalized $\mathrm{Fe}^{+}\left(h_{1 D}^{*}\right)$ configuration.

level, however, has a strong $p$ character due to one of the surrounding $N$ atoms holding a small polaron, as revealed by the density of states shown in Figs. 1(a) and 1(b) and the real-space charge density distribution given in Figs. 2(a) and 2(b). This result points to a suitable explanation for $\mathrm{Mn}$ dopants in $\mathrm{GaN}$ based on $d^{5}$ plus a localized polaron. For $\mathrm{Fe}^{+}$, the lowest unoccupied state resembles that of $\mathrm{Mn}^{0}$-it is nondegenerate, strongly localized on one of the surrounding $N$ atoms, and accompanied by a symmetry-breaking structural relaxation, thus best described as a small polaron state [see Figs. 1(c), 1(d), 2(c), and 2(d)]. Hence, both $\mathrm{Mn}^{0}$ and $\mathrm{Fe}^{+}$exhibit $d^{5}+h$ ground states with the $\mathrm{N} p$ hole tightly bound to the transition metal impurity, regardless of whether the occupied transition metal $d$ levels are in the gap or below $E_{v}$.

Notice that for $\mathrm{Mn}$ the ground state polaron is along the [0001] direction (wurtzite $c$ axis), but for $\mathrm{Fe}^{+}$the ground state polaron is along one of the $\langle 1 \overline{1} 01\rangle$ directions (bond axis with one of the $\mathrm{N}$ atoms in the $a b$ plane). These two differently oriented hole polarons are henceforth denoted by $h_{c}$ and $h_{a b}$, respectively. Using this notation, the $\mathrm{Fe}^{+}$ground state configuration is $d^{5}+h_{a b}$ and the Mn ground state configuration is $d^{5}+h_{c}$. However, for both impurities, both small polaron states are available: $\mathrm{Fe}^{+}$has a $d^{5}+h_{c}$ configuration $25 \mathrm{meV}$ 
(a) $\mathrm{Mn}^{0}\left(d^{5}+h_{c}\right)$ top view

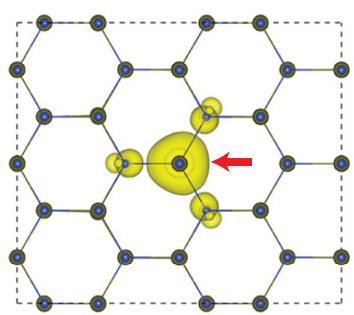

(c) $\mathrm{Fe}^{+}\left(d^{5}+h_{a b}\right)$ top view

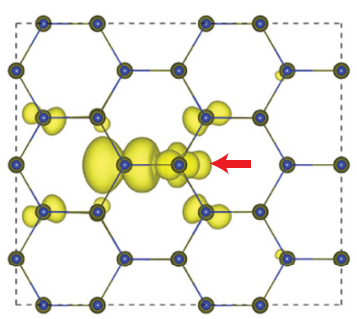

(e) $\mathrm{Fe}^{+}\left(d^{5}+h_{I D}^{*}\right)$ top view

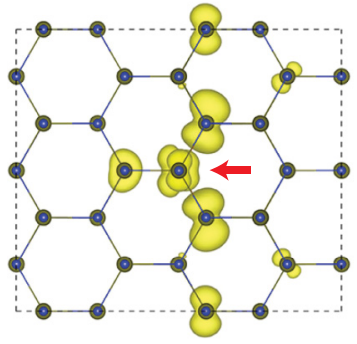

(b) $\mathrm{Mn}^{0}\left(d^{5}+h_{c}\right)$ side view

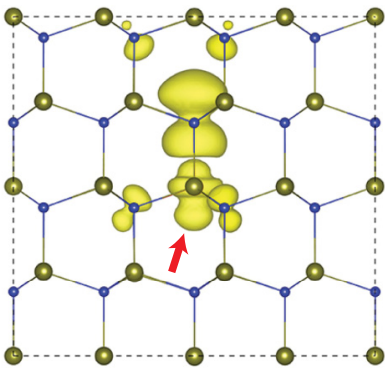

(d) $\mathrm{Fe}^{+}\left(d^{5}+h_{a b}\right)$ side view

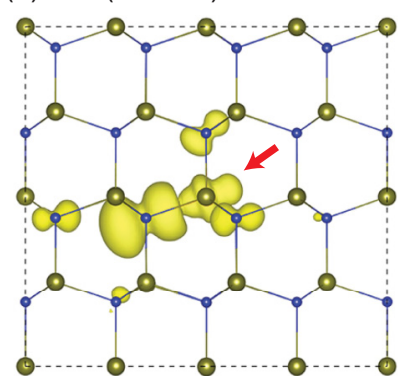

(f) $\mathrm{Fe}^{+}\left(d^{5}+h_{l D}^{*}\right)$ side view

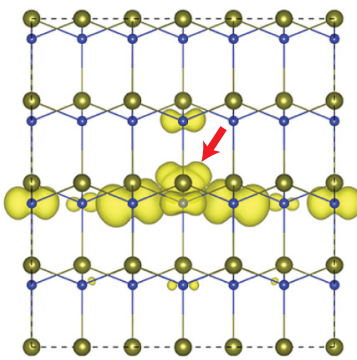

FIG. 2. Charge densities of the lowest unoccupied states, i.e., hole states around the dopant for the $\mathrm{Mn}^{0}\left(d^{5}+h_{c}\right), \mathrm{Fe}^{+}\left(h_{a b}^{*}\right)$, and $\mathrm{Fe}^{+}$ $\left(h_{1 D}^{*}\right)$ configurations calculated in the 96 atom supercell (isosurface density: $0.02 \mathrm{e} / \AA$ ). The central transition metal dopant is indicated by a red arrow. The top views in panels (a), (c), and (e) show the supercell from the [0001] direction, the side views in panels (b) and (d) from the [1120] direction, and the side view in panel (f) from the [1100] direction.

above the ground state and Mn a $d^{5}+h_{a b}$ configuration $14 \mathrm{meV}$ above the ground state. This finding suggests that there may be other stable configurations with different hole states.

\section{MULTIFARIOUS HOLE STATES}

For $\mathrm{Fe}^{+}$, in addition to the polaron ground state, we find a delocalized partially occupied dangling-bond hybrid (DBH) like state $\left(\mathrm{Fe}^{+}\right)^{*}$, akin to the one suggested earlier as the ground state [39-41]. The system exhibits a bistability because this $\left(\mathrm{Fe}^{+}\right)^{*}$ configuration is only $37 \mathrm{meV}$ less stable than the polaronic ground state. This delocalized quasi-1D state $\left(h_{1 \mathrm{D}}^{*}\right)$ spreads over one of the six equivalent $\langle 11 \overline{2} 0\rangle$ directions [see Figs. 2(e) and 2(f)], chosen randomly.

For $\mathrm{Mn}^{0}$, such a $h_{1 \mathrm{D}}^{*}$ state appears by increasing the impurity concentration to $\sim 2.8 \%$, i.e., calculating the system in a 72

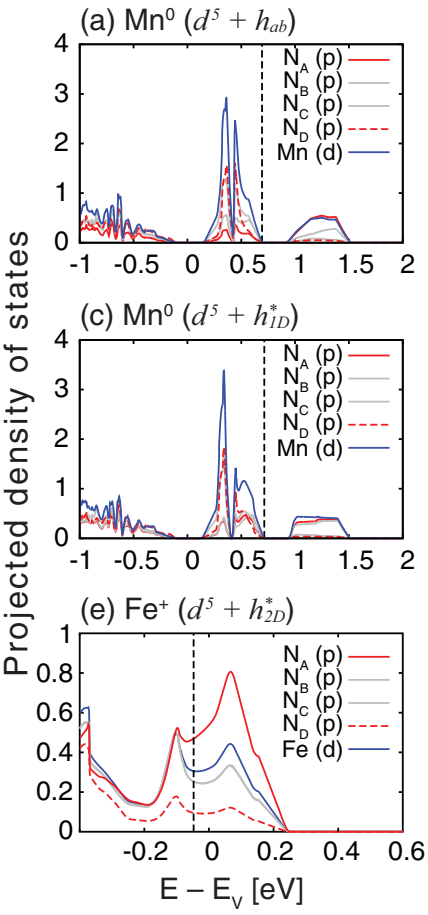

(b) $\mathrm{Mn}^{0}\left(d^{5}+h_{a b}\right)$ top view

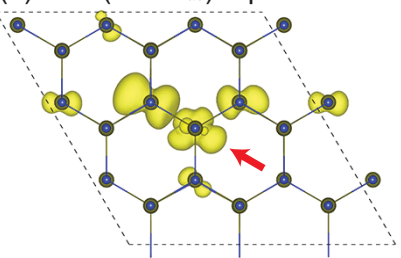

(d) $\mathrm{Mn}^{0}\left(d^{5}+h_{I D}^{*}\right)$ top view

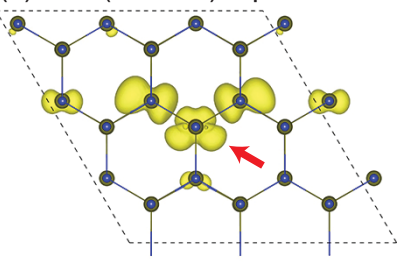

(f) $\mathrm{Fe}^{+}\left(d^{5}+h_{2 D}^{*}\right)$ top view

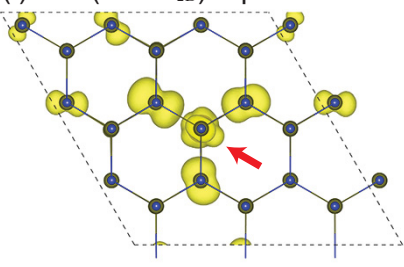

FIG. 3. Different hole states calculated in 72 atom supercell. Panels (a), (c), and (e) show projected densities of states for $\mathrm{Mn}^{0}$ $\left(d^{5}+h_{a b}\right)$ polaron ground state, $\mathrm{Mn}^{0}\left(h_{1 D}^{*}\right)$ extended state, and $\mathrm{Fe}^{+}$ $\left(h_{2 D}^{*}\right)$ extended state, respectively. Panels (b), (d), and (f) show hole densities around central $\mathrm{Mn}$ atom for $\mathrm{Mn}^{0}\left(d^{5}+h_{a b}\right), \mathrm{Mn}^{0}\left(h_{1 D}^{*}\right)$, and $\mathrm{Fe}^{+}\left(h_{2 D}^{*}\right)$, respectively, viewed from the [0001] direction (isosurface density: $0.02 \mathrm{e} / \AA$ ).

atom supercell. At this higher concentration, however, the ground state has a $h_{a b}$ polaron, the $h_{c}$ polaron is less stable by $33 \mathrm{meV}$, and the extended $h_{1 \mathrm{D}}^{*}$ state is even less stable by $61 \mathrm{meV}$ [density of states and hole charge density shown in Figs. 3(a)-3(d)]. The relative energies are summarized in Table I. The fact that the impurity-to-impurity distance in the [1120] direction is exactly the same in both the 96 and 72 atom supercells suggests that this extended state is not simply a supercell effect [42], or a plurality effect [43] triggered by the presence of inactive spectator images, but indicates an insulator-metal transition when the Mn concentration is further increased [8]. Indeed, the hole charge density in the 72 atom supercell for the small polaron states $\left[h_{a b}\right.$ shown in Figs. 3(b)] is more extended than the polaron states calculated in the 96 atom supercells [Figs. 2(a)-2(b)].

$\mathrm{Fe}^{+}$calculated in the 72 atom supercell exhibits even more rich behavior. In addition to the $h_{a b}, h_{c}$, and $\left(h_{1 \mathrm{D}}^{*}\right)$ states, we find another delocalized state $\left[h_{2 \mathrm{D}}^{*}\right.$, shown in Figs. 3(e)-3(f)], which is extended in the wurtzite $a b$ plane. The $h_{a b}$ small polaron state is more stable, but the $h_{c}$ and $h_{1 \mathrm{D}}^{*}$ states (nearly degenerate in energy) are 18 and $19 \mathrm{meV}$ less stable than the ground state (relative energies summarized in Table I). The emergence of delocalized states also for $\mathrm{Fe}^{+}$upon increasing impurity concentration evidences that $\mathrm{Mn}$ and $\mathrm{Fe}$ doped magnetic semiconductor systems are in the vicinity of an insulator-to-metal transition. However, as concentration further increases, clustering effects are expected to trap carrier holes [4-6], and/or, the system may phase separate [44-47] 
TABLE I. Relative stability of various hole states under axial strain. $h_{a b}$ and $h_{c}$ are polaronic hole states, and, $h_{1 \mathrm{D}}^{*}$ and $h_{2 \mathrm{D}}^{*}$ are extended states. Uniaxial strain ( + for tensile, - for compressive) along the $c$ axis is given by $\delta_{c}$, and the associated perpendicular lattice distortion by $\gamma_{a b}$. The ground state configuration for each strain condition is set as $E=0$, and the relative energies of higher energy configurations are given in $\mathrm{meV}$. All data is calculated in 72 atom supercells.

\begin{tabular}{|c|c|c|c|c|c|}
\hline$\delta_{c}$ & $\gamma_{a b}$ & $E\left(h_{1 \mathrm{D}}^{*}\right)$ & $E\left(h_{2 \mathrm{D}}^{*}\right)$ & $E\left(h_{a b}\right)$ & $E\left(h_{c}\right)$ \\
\hline \multicolumn{6}{|c|}{ GaN:Mn } \\
\hline$-10 \%$ & $2.80 \%$ & & & & 0 \\
\hline$-7 \%$ & $1.83 \%$ & & & & 0 \\
\hline$-5 \%$ & $1.28 \%$ & & & & 0 \\
\hline$-3 \%$ & $0.81 \%$ & & & & 0 \\
\hline$-1 \%$ & $0.39 \%$ & & & 16 & 0 \\
\hline \multicolumn{2}{|c|}{ unstrained } & 61 & & 0 & 33 \\
\hline $1 \%$ & $0.02 \%$ & & & 0 & 71 \\
\hline $3 \%$ & $-0.28 \%$ & 50 & & 0 & \\
\hline $5 \%$ & $-0.72 \%$ & 61 & & 0 & \\
\hline $7 \%$ & $-0.72 \%$ & 58 & & 0 & \\
\hline $10 \%$ & $-0.89 \%$ & 63 & & 0 & \\
\hline \multicolumn{6}{|c|}{$\overline{\mathrm{GaN}: \mathrm{Fe}}$} \\
\hline$-10 \%$ & $2.80 \%$ & & & & 0 \\
\hline$-7 \%$ & $1.83 \%$ & & & & 0 \\
\hline$-5 \%$ & $1.28 \%$ & & 182 & & 0 \\
\hline$-3 \%$ & $0.81 \%$ & 71 & 92 & 80 & 0 \\
\hline$-1 \%$ & $0.39 \%$ & & 10 & 15 & 0 \\
\hline \multicolumn{2}{|c|}{ unstrained } & 19 & 43 & 0 & 18 \\
\hline $1 \%$ & $0.02 \%$ & & 0 & 20 & \\
\hline $3 \%$ & $-0.28 \%$ & & 0 & & \\
\hline $5 \%$ & $-0.72 \%$ & & 0 & 21 & \\
\hline $7 \%$ & $-0.72 \%$ & & 0 & 14 & \\
\hline $10 \%$ & $-0.89 \%$ & & 3 & 0 & \\
\hline
\end{tabular}

before exhibiting a Mott transition like Mn-doped GaAs [8]. Therefore it is desirable to find other means to stabilize the extended/delocalized hole states.

\section{STRAIN CONTROL OF HOLE LOCALIZATION}

$\mathrm{GaN}$ is a piezoelectric, so axial strain causes electric polarization $\boldsymbol{P}_{\mathrm{s}}$, as illustrated in Fig. 4. For example, [0001] tensile strain displaces negative charge along the positive $c$ axis, leading to polarization $\boldsymbol{P}_{\mathrm{s}} \| c$. The small polaron $d^{5}+h$ systems imply a displacement of positive charge from three anions to the fourth neighbor of the $d^{5}$ center, which for the $h_{c}$ polaron implies a polarization $\boldsymbol{P}_{\mathrm{p}} 1 \uparrow c$. Thus, an $h_{c}$ polaron in a GaN host under [0001] tensile strain exhibits opposite polarization than the surroundings, i.e., $\boldsymbol{P}_{\mathrm{s}} \| \boldsymbol{P}_{\mathrm{p}}$, which may lead to some destabilization as, in general, polarized systems have the tendency to align their polarizations.

We test this by applying such tensile strain for the defective systems (shown in Table I), and indeed, for both $\mathrm{Mn}$ or $\mathrm{Fe}^{+}$ impurities, tensile strain in the [0001] direction destabilizes the $h_{c}$ polaron, and compressive strain stabilizes the $h_{c}$ polaron. For Mn-doped GaN, the tensile strain stabilizes $h_{a b}$ polaron, while for $\mathrm{Fe}^{+}$, the extended $h_{2 \mathrm{D}}^{*}$ hole state becomes

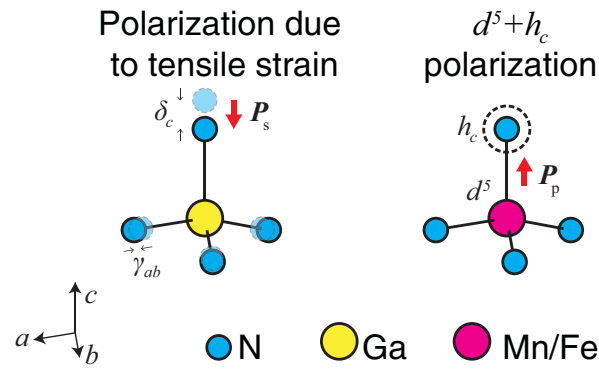

FIG. 4. Polarization due to strain $\boldsymbol{P}_{\mathrm{s}}$ and $d^{5}+h_{c}$ polaron state $\boldsymbol{P}_{\mathrm{p}}$. Tensile [0001] direction strain (left panel), shifts the negative anions along the positive $c$ axis, i.e., $\boldsymbol{P}_{\mathrm{s}} \| \boldsymbol{c} . d^{5}+h_{c}$ ) small polaron formation shifts positive charge along the positive $c$ axis, i.e., $\boldsymbol{P}_{\mathrm{p}} \Uparrow c$.

the ground state. Thus, the relative stability of these two competing phases may be controlled by axial strain, due to, e.g., interfaces [48] or substrates [49]. The strain control of hole states being associated to electric polarization suggests that the hole localization/delocalization in these systems can equally well be controlled by electric gating. In fact, such intertwining of the piezoelectric effect and magnetic interactions has already been observed [23].

\section{DISCUSSION}

We have shown that $\mathrm{Mn}^{0}$ and $\mathrm{Fe}^{+}$dopants in $\mathrm{GaN}$ have polaronic $d^{5}+h$ ground states with holes trapped by one of the neighboring $\mathrm{N}$ atoms. However, we also find extended hole states slightly less stable than the polaronic ground states, and thus, the holes around the magnetic $d^{5}$ impurities exhibit multifarious behavior. Extended and trapped carrier states are associated with completely different magnetic interactions, so this multifarious behavior must be included in any attempt to describe the elusive, yet strong magnetic interactions observed in magnetic semiconductor materials.

The multifarious hole states can be engineered via axial strain, i.e., the relative stability of the different hole states can be controlled by varying strain conditions by interface [48] or substrate strain [49]. Compressive uniaxial strain along the [0001] direction stabilizes polarons oriented along the same [0001] direction, whereas tensile [0001] strain stabilizes polarons within the (0001) plane for Mn-doped GaN, and, extended hole states in the (0001) plane for Fe-doped GaN. The axial strain and different hole states are intertwined by the piezoelectric effect, such that strain causes electric polarization of $\mathrm{GaN}$, which in turn affects the preferable direction of the polaron dipole moment, or destabilizes such a polaron state altogether. By the same token, the multifarious hole states can be controlled by external gating (inverse piezoelectric effect), as indeed recently was observed experimentally [23].

For $\mathrm{Mn}$-doped $\mathrm{GaN}$, the unstrained polaron ground state is unlikely to foster long-range ferromagnetic interactions, as evidenced by cryogenic Curie temperatures of around $10 \mathrm{~K}$ $[17,22,23]$. However, Fe-doped GaN has extended hole states under tensile uniaxial strain conditions similar as may be expected on GaN samples deposited on sapphire substrates [49]. This strain stabilization of the extended hole states happens at high impurity concentrations in the vicinity of a Mott 
transition [8]. Thus, suitable strain conditions and a sufficiently high impurity concentration may lead to much higher Curie temperatures even for Mn-doped $\mathrm{GaN}$, which puts in new light the extraordinary high Curie temperature of $940 \mathrm{~K}$ observed in highly strained thin-film samples of Mn-doped $\mathrm{GaN}$ [21]. Because the strain induced transition from localized to delocalized states mostly involves host states, similar transitions may also occur for $\mathrm{Mg}\left(d^{0}\right)$, Be $\left(d^{0}\right)$ or $\mathrm{Zn}\left(d^{10}\right)$ impurities in the same host [35-38], or, $\left(d^{0}\right)$ or $\left(d^{10}\right)$ impurities in oxides. This strain stabilization of extended states may even offer an explanation to the phantom ferromagnetism in thin-film oxides [50]. In the respective bulk materials, carriers tend to be trapped in polaron states [35-38] but may well become delocalized to facilitate ferromagnetic interactions due to substrate strain in thin-film samples.

\section{ACKNOWLEDGMENTS}

We thank T. Dietl, S. Lany, and A. Zunger for many valuable discussions, as well as D. Lamoen and O. Schirmer for their comments on the manuscript. This work was funded by JSPS Grant-in-Aid for Scientific Research (C) No. 17K05494. H.R. is grateful to the São Paulo Research Foundation FAPESP and S.B. thanks the Rotary Yoneyama Memorial Foundation for support. A.A. acknowledges support of the Project FIS201676617-P of the Spanish Ministry of Economy and Competitiveness MINECO.C.E.-A. acknowledges the hospitality of the Donostia International Physics Center. Numerical calculations were performed at the Research Center for Computational Science, Okazaki and at ISSP, The University of Tokyo.

\section{APPENDIX: ELECTRONIC TRANSITIONS}

Both $\mathrm{Mn}^{0}$ and $\mathrm{Fe}^{+}$are assumed to be stable under $p$ type doping, i.e., with the Fermi level in the lower part of the band gap. Thus we focus on the electronic transitions close to the valence band and calculate the thermal transition energies $\varepsilon_{\mathrm{T}}(q / q-1)=E(q-1)-E(q)$ (Table II). For Mn, we also evaluate the optical transition energies in the Franck-Condon approximation [51-53], $\varepsilon_{\mathrm{FC}}(q / q-1)=E(q-1 ; q)-E(q)$, where $E(q)$ is the total energy for charge state $q$, and $E(q-$ $1 ; q)$ refers to charge state $q-1$ in the fixed geometry of $q$. Supercell size effects in total energies are treated as described in Refs. [54-56], including a rigid shift of the valence band by $0.27 \mathrm{eV}$ [57] for transitions involving a CFR state. For Fe, the $(+/ 0)$ transition lies slightly below the valence band edge, which means it requires degenerate hole doping of $10^{21} \mathrm{~cm}^{-3}$ to be stabilized, which is larger than typically achieved in p-type GaN.

\section{Comparison of GaN:Mn with optical experiment}

The optical properties of GaN:Mn are well known-there are two absorption features at around 1.5 and $1.8 \mathrm{eV}$ associated with $\mathrm{Mn}$ acceptor transitions. The band at $1.8 \mathrm{eV}$ is
TABLE II. Calculated thermal and optical (Franck-Condon) transition energies $\varepsilon_{\mathrm{T}}(q / q-1)$ and $\varepsilon_{\mathrm{FC}}(q / q-1)$, given in $\mathrm{eV}$, for $\mathrm{Mn}$ and Fe dopants in $\mathrm{GaN}$, for both the polaronic ground state and the (higher energy) delocalized state.

\begin{tabular}{lcc}
\hline \hline & polaron GS & delocalized state \\
\hline GaN:Mn (96 atom supercell) & \\
$\varepsilon_{\mathrm{T}}(+/ 0)$ & 0.58 & \\
$\varepsilon_{\mathrm{FC}}(+/ 0)$ & 1.29 & \\
$\varepsilon_{\mathrm{T}}(0 /-)$ & 1.40 & \\
$\varepsilon_{\mathrm{FC}}(0 /-)$ & 1.69 & \\
\hline $\mathrm{GaN}: \mathrm{Mn}(72$ atom supercell) & \\
$\varepsilon_{\mathrm{T}}(+/ 0)$ & 0.57 & 1.38 \\
$\varepsilon_{\mathrm{FC}}(+/ 0)$ & 1.18 & 1.63 \\
$\varepsilon_{\mathrm{T}}(0 /-)$ & 1.44 & \\
$\varepsilon_{\mathrm{FC}}(0 /-)$ & 1.69 & -0.14 \\
\hline $\mathrm{GaN}: \mathrm{Fe}(96$ atom supercell $)$ & \\
$\varepsilon_{\mathrm{T}}(+/ 0)$ & -0.10 & \\
\hline \hline
\end{tabular}

commonly attributed to the (0/-) acceptor transition [58-60]. The other feature, which in fact has multiple lines around $1.5 \mathrm{eV}$, starting from a peak at $1.42 \mathrm{eV}$, was also initially attributed to the $(0 /-)$ acceptor transition $[61,62]$ and later reinterpreted to be due to a $\mathrm{Mn}$ internal transition, to occur between either $e$ and $t$ levels [58], or the symmetry broken Mn CFR levels $[9,10]$. Typically, experimental absorption features are compared with optical transitions calculated in the FranckCondon approximation, and indeed, $\varepsilon_{\mathrm{FC}}(0 /-)=1.69 \mathrm{eV}$ for $\mathrm{Mn}$, as given in Table II, is well in agreement with the experimental feature observed at $1.8 \mathrm{eV}$. The metastable delocalized state found for $\mathrm{Mn}$ in the 72 atom supercell (Mn concentration of $2.8 \%$ ) has a Franck-Condon transition only slightly lower in energy, $\varepsilon_{\mathrm{FC}}(0 /-)=1.63 \mathrm{eV}$, which cannot be linked to the broad absorption peak at $1.5 \mathrm{eV}$. The $\varepsilon_{\mathrm{FC}}(+/ 0)=1.29 \mathrm{eV}$ transition [63] may offer an interpretation for the $1.5 \mathrm{eV}$ peak. However, it is questionable whether or not this $(+/ 0)$ transition could yield that high intensities and the temperature activated changes observed in photocurrents [58]. Another interpretation for the $1.5 \mathrm{eV}$ peak comes from the fact that the Franck-Condon approximation breaks down for polaron states $[64,65]$. As a consequence, in addition to the Franck-Condon spectrum of minimal kinetic energy [51-53], a sharp absorption peak may appear at the thermodynamic transition $\varepsilon_{T}$ (maximal kinetic energy [66]), here calculated as $\varepsilon_{\mathrm{T}}(0 /-)=1.4 \mathrm{eV}$ (see Table II), in good agreement with the experimental $1.5 \mathrm{eV}$ peak. The obtained difference between $\varepsilon_{F C}$ and $\varepsilon_{T}, 0.29 \mathrm{eV}$, is the relaxation energy, or the thermal barrier required to activate the photocurrent at the $\varepsilon_{T}$ peak. It approaches the experimental value of $0.3 \mathrm{eV}$ and also is comparable to the Stokes shift calculated in other Mn-doped systems such as nanocrystals [67].
[1] A. Zunger, S. Lany, and H. Raebiger, The quest for dilute ferromagnetism in semiconductors: Guides and misguides by theory, Physics 3, 53 (2010).
[2] T. Dietl and H. Ohno, Dilute ferromagnetic semiconductors: Physics and spintronic structures, Rev. Mod. Phys. 86, 187 (2014). 
[3] M. Tanaka, S. Ohya, and P. Nam Hai, Recent progress in III-V based ferromagnetic semiconductors: Band structure, Fermi level, and tunneling transport, Appl. Phys. Rev. 1, 011102 (2014).

[4] H. Raebiger, A. Ayuela, and R. Nieminen, Intrinsic hole localization mechanism in magnetic semiconductors, J. Phys.: Condens. Matter 16, L457 (2004).

[5] T. Hynninen, H. Raebiger, and J. von Boehm, A multiscale study of ferromagnetism in clustered $(\mathrm{Ga}, \mathrm{Mn}) \mathrm{N}$, J. Phys.: Condens. Matter 18, 1561 (2006).

[6] T. Hynninen, M. Ganchenkova, H. Raebiger, and J. von Boehm, Ferromagnetism and its evolution during long-term annealing in (Ga,Mn)As, Phys. Rev. B 74, 195337 (2006).

[7] H. Raebiger, S. Lany, and A. Zunger, Charge self-regulation upon changing the oxidation state of transition metals in insulators, Nature (London) 453, 763 (2008).

[8] S. Bae and H. Raebiger, Critical metal-insulator transition due to nuclear quantum effects in Mn-doped GaAs, Phys. Rev. B 94, 241115 (2016).

[9] A. Stroppa and G. Kresse, Unraveling the Jahn-Teller effect in Mn-doped GaN using the Heyd-Scuseria-Ernzerhof hybrid functional, Phys. Rev. B 79, 201201 (2009).

[10] F. Virot, R. Hayn, and A. Boukortt, Electronic structure and Jahn-Teller effect in GaN:Mn and ZnS:Cr, J. Phys.: Condens. Matter 23, 025503 (2010).

[11] F. C. Zhang and T. M. Rice, Effective Hamiltonian for the superconducting Cu oxides, Phys. Rev. B 37, 3759 (1988).

[12] A. Filippetti, N. A. Spaldin, and S. Sanvito, Self-interaction effects in (Ga, Mn) As and (Ga, Mn) N, Chem. Phys. 309, 59 (2004).

[13] T. Dietl, F. Matsukura, and H. Ohno, Ferromagnetism of magnetic semiconductors: Zhang-Rice limit, Phys. Rev. B 66, 033203 (2002).

[14] T. Dietl, Hole states in wide band-gap diluted magnetic semiconductors and oxides, Phys. Rev. B 77, 085208 (2008).

[15] R. Nelson, T. Berlijn, J. Moreno, M. Jarrell, and W. Ku, What is the Valence of $\mathrm{Mn}$ in $\mathrm{Ga}_{1-x} \mathrm{Mn}_{x} \mathrm{~N}$ ?, Phys. Rev. Lett. 115, 197203 (2015).

[16] W. Stefanowicz, D. Sztenkiel, B. Faina, A. Grois, M. Rovezzi, T. Devillers, F. d'Acapito, A. Navarro-Quezada, T. Li, R. Jakieła, M. Sawicki, T. Dietl, and A. Bonanni, Structural and paramagnetic properties of dilute $\mathrm{Ga}_{1-x} \mathrm{Mn}_{x} \mathrm{~N}$, Phys. Rev. B 81, 235210 (2010).

[17] A. Bonanni, M. Sawicki, T. Devillers, W. Stefanowicz, B. Faina, T. Li, T. E. Winkler, D. Sztenkiel, A. Navarro-Quezada, M. Rovezzi, R. Jakieła, A. Grois, M. Wegscheider, W. Jantsch, J. Suffczyński, F. d'Acapito, A. Meingast, G. Kothleitner, and T. Dietl, Experimental probing of exchange interactions between localized spins in the dilute magnetic insulator $(\mathrm{Ga}, \mathrm{Mn}) \mathrm{N}$, Phys. Rev. B 84, 035206 (2011).

[18] E. Malguth, A. Hoffmann, and M. R. Phillips, Fe in III-V and II-VI semiconductors, Phys. Status Solidi B 245, 455 (2008).

[19] A. Navarro-Quezada, N. Gonzalez Szwacki, W. Stefanowicz, T. Li, A. Grois, T. Devillers, M. Rovezzi, R. Jakieła, B. Faina, J. A. Majewski, M. Sawicki, T. Dietl, and A. Bonanni, Fe-Mg interplay and the effect of deposition mode in $(\mathrm{Ga}, \mathrm{Fe}) \mathrm{N}$ doped with Mg, Phys. Rev. B 84, 155321 (2011).

[20] P. Alippi, F. Filippone, G. Mattioli, A. A. Bonapasta, and V. Fiorentini, Bound states of the Fe impurity in wurtzite GaN from hybrid density-functional calculations, Phys. Rev. B 84, 033201 (2011).

[21] T. Sasaki, S. Sonoda, Y. Yamamoto, K. Suga, S. Shimizu, K. Kindo, and H. Hori, Magnetic and transport characteristics on high Curie temperature ferromagnet of Mn-doped GaN, J. Appl. Phys. 91, 7911 (2002).

[22] M. Overberg, C. Abernathy, S. Pearton, N. Theodoropoulou, K. McCarthy, and A. Hebard, Indication of ferromagnetism in molecular-beam-epitaxy-derived N-type GaMnN, Appl. Phys. Lett. 79, 1312 (2001).

[23] D. Sztenkiel, M. Foltyn, G. P. Mazur, R. Adhikari, K. Kosiel, K. Gas, M. Zgirski, R. Kruszka, R. Jakiela, T. Li, A. Piotrowska, A. Bonanni, M. Sawicki, and T. Dietl, Stretching magnetism with an electric field in a nitride semiconductor, Nat. Commun. 7, 13232 (2016).

[24] V. Ivády, I. A. Abrikosov, E. Janzen, and A. Gali, Role of screening in the density functional applied to transitionmetal defects in semiconductors, Phys. Rev. B 87, 205201 (2013).

[25] S. Lany, Band-structure calculations for the $3 \mathrm{~d}$ transition metal oxides in G W, Phys. Rev. B 87, 085112 (2013).

[26] F. Da Pieve, S. Di Matteo, T. Rangel, M. Giantomassi, D. Lamoen, G. M. Rignanese, and X. Gonze, Origin of Magnetism and Quasiparticles Properties in Cr-Doped TiO2, Phys. Rev. Lett. 110, 136402 (2013).

[27] A. Boukortt, R. Hayn, and F. Virot, Optical properties of Mndoped GaN, Phys. Rev. B 85, 033302 (2012).

[28] S. Lany and A. Zunger, Polaronic hole localization and multiple hole binding of acceptors in oxide wide-gap semiconductors, Phys. Rev. B 80, 085202 (2009).

[29] S. Lany, Predicting polaronic defect states by means of generalized Koopmans density functional calculations, Phys. Status Solidi B 248, 1052 (2011).

[30] P. E. Blöchl, Projector augmented-wave method, Phys. Rev. B 50, 17953 (1994).

[31] G. Kresse and D. Joubert, From ultrasoft pseudopotentials to the projector augmented-wave method, Phys. Rev. B 59, 1758 (1999).

[32] H. Raebiger, S. Lany, and A. Zunger, Electronic structure, donor and acceptor transitions, and magnetism of $3 \mathrm{~d}$ impurities in $\mathrm{In}_{2} \mathrm{O}_{3}$ and $\mathrm{ZnO}$, Phys. Rev. B 79, 165202 (2009).

[33] K. Sato, J. Kudrnovský, P. H. Dederichs, O. Eriksson, I. Turek, B. Sanyal, G. Bouzerar, H. Katayama-Yoshida, V. A. Dinh, T. Fukushima, H. Kizaki, and R. Zeller, First-principles theory of dilute magnetic semiconductors, Rev. Mod. Phys. 82, 1633 (2010).

[34] M. Farzalipour Tabriz, B. Aradi, T. Frauenheim, and P. Deák, Application of the Lany-Zunger polaron correction for calculating surface charge trapping, J. Phys.: Condens. Matter 29, 394001 (2017).

[35] A. Droghetti, C. D. Pemmaraju, and S. Sanvito, Predicting $d^{0}$ magnetism: Self-interaction correction scheme, Phys. Rev. B 78, 140404 (2008).

[36] S. Lany and A. Zunger, Dual nature of acceptors in GaN and $\mathrm{ZnO}$ : The curious case of the shallow Mg Ga deep state, Appl. Phys. Lett. 96, 142114 (2010).

[37] J. L. Lyons, A. Janotti, and C. G. Van de Walle, Shallow versus Deep Nature of Mg Acceptors in Nitride Semiconductors, Phys. Rev. Lett. 108, 156403 (2012). 
[38] O. F. Schirmer, On the coexistence of localized and extended acceptor states in high gap semiconductors, Semicond. Sci. Technol. 30, 024009 (2015).

[39] K. Sato and H. Katayama-Yoshida, Material design of GaNbased ferromagnetic diluted magnetic semiconductors, Jpn. J. Appl. Phys. 40, L485 (2001).

[40] P. Mahadevan and A. Zunger, First-principles investigation of the assumptions underlying model-Hamiltonian approaches to ferromagnetism of $3 \mathrm{~d}$ impurities in III-V semiconductors, Phys. Rev. B 69, 115211 (2004).

[41] Y.-J. Zhao, P. Mahadevan, and A. Zunger, Practical rules for orbital-controlled ferromagnetism of $3 \mathrm{~d}$ impurities in semiconductors, J. Appl. Phys. 98, 113901 (2005).

[42] Notice that supercell effects due to image charges have been corrected for as described in Refs. [54-56].

[43] A. Franceschetti, S. V. Dudiy, S. V. Barabash, A. Zunger, J. $\mathrm{Xu}$, and $\mathrm{M}$. van Schilfgaarde, First-Principles Combinatorial Design of Transition Temperatures in Multicomponent Systems: The Case of Mn in GaAs, Phys. Rev. Lett. 97, 047202 (2006).

[44] H. Raebiger, M. Ganchenkova, and J. von Boehm, Diffusion and clustering of substitutional $\mathrm{Mn}$ in (Ga,Mn)As, Appl. Phys. Lett. 89, 012505 (2006).

[45] H. Raebiger, A. Ayuela, J. von Boehm, and R. Nieminen, Clustering of Mn in (Ga,Mn)As, J. Magn. Magn. Mater. 290291, 1398 (2005).

[46] H. Raebiger, H. Nakayama, and T. Fujita, Control of defect binding and magnetic interaction energies in dilute magnetic semiconductors by charge state manipulation, J. Appl. Phys. 115, 012008 (2014).

[47] T. Fujita and H. Raebiger, Pairwise chemical interactions of charged transition-metal impurities in insulators, Phys. Rev. B 90, 161201 (2014).

[48] T. Kagawa and H. Raebiger, Schottky Barrier Formation and Strain at the (011) GdN/GaN Interface from First Principles, Phys. Rev. Appl. 2, 054009 (2014).

[49] H. Amano, K. Hiramatsu, and I. Akasaki, Heteroepitaxial Growth and the Effect of Strain on the Luminescent Properties of GaN Films on (11 20) and (0001) Sapphire Substrates, Jpn. J. Appl. Phys. 27, L1384 (1988).

[50] J. Coey, P. Stamenov, and R. D. Gunning, Ferromagnetism in defect-ridden oxides and related materials, New J. Phys. 12, 053025 (2010).

[51] J. Franck and E. G. Dymond, Elementary processes of photochemical reactions, Trans. Faraday Soc. 21, 536 (1926).
[52] E. Condon, A theory of intensity distribution in band systems, Phys. Rev. 28, 1182 (1926).

[53] E. Condon, Nuclear motions associated with electron transitions in diatomic molecules, Phys. Rev. 32, 858 (1928).

[54] C. Persson, Y. J. Zhao, S. Lany, and A. Zunger, n-type doping of CuInSe2 and CuGaSe2, Phys. Rev. B 72, 035211 (2005).

[55] S. Lany and A. Zunger, Assessment of correction methods for the band-gap problem and for finite-size effects in supercell defect calculations: Case studies for $\mathrm{ZnO}$ and GaAs, Phys. Rev. B 78 , 235104 (2008).

[56] S. Lany and A. Zunger, Many-body GW calculation of the oxygen vacancy in ZnO, Phys. Rev. B 81, 113201 (2010).

[57] H. Raebiger, Theory of defect complexes in insulators, Phys. Rev. B 82, 073104 (2010).

[58] T. Graf, M. Gjukic, M. S. Brandt, M. Stutzmann, and O. Ambacher, The $\mathrm{Mn}[\sup 3+/ 2+]$ acceptor level in group III nitrides, Appl. Phys. Lett. 81, 5159 (2002).

[59] A. Wolos, M. Palczewska, M. Zajac, J. Gosk, M. Kamińska, A. Twardowski, M. Bockowski, I. Grzegory, and S. Porowski, Optical and magnetic properties of Mn in bulk GaN, Phys. Rev. B 69, 115210 (2004).

[60] A. Wolos, A. Wysmolek, M. Kamińska, A. Twardowski, M. Bockowski, I. Grzegory, S. Porowski, and M. Potemski, Neutral Mn acceptor in bulk GaN in high magnetic fields, Phys. Rev. B 70, 245202 (2004)

[61] R. Y. Korotkov, J. M. Gregie, and B. W. Wessels, Mn-related absorption and PL bands in $\mathrm{GaN}$ grown by metal organic vapor phase epitaxy, Physica B 308-310, 30 (2001).

[62] R. Y. Korotkov, J. M. Gregie, and B. W. Wessels, Optical properties of the deep $\mathrm{Mn}$ acceptor in GaN:Mn, Appl. Phys. Lett. 80, 1731 (2002).

[63] Note that $\mathrm{Mn}^{+}$has a $d^{5}+2 h$ bipolaron configuration and this is a bipolaron to polaron transition.

[64] J. Devreese, J. De Sitter, and M. Goovaerts, Optical absorption of polarons in the feynman-hellwarth-iddings-platzman approximation, Phys. Rev. B 5, 2367 (1972).

[65] G. De Filippis, V. Cataudella, A. S. Mishchenko, C. A. Perroni, and J. T. Devreese, Validity of the Franck-Condon Principle in the Optical Spectroscopy: Optical Conductivity of the Fröhlich Polaron, Phys. Rev. Lett. 96, 136405 (2006).

[66] R. S. Mulliken, Role of kinetic energy in the franck-condon principle, J. Chem. Phys. 55, 309 (1971).

[67] C. Echeverría-Arrondo, J. Pérez-Conde, and A. Ayuela, Optical spin control in nanocrystalline magnetic nanoswitches, Appl. Phys. Lett. 95, 043111 (2009). 